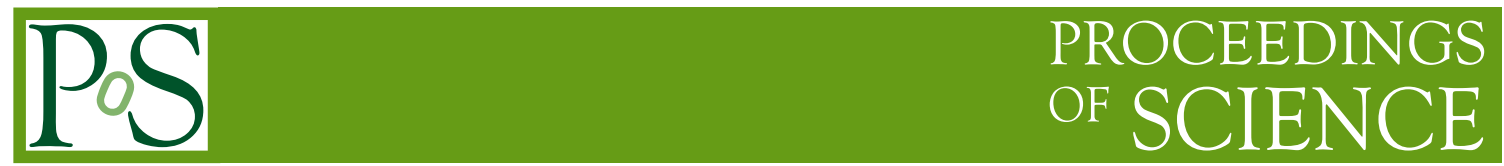

\title{
Multi-frequency Behaviour of Nova-like Systems
}

\author{
Polina Zemko* \\ Department of Physics and Astronomy, Universitá di Padova, vicolo dell' Osservatorio 3, \\ I-35122 Padova, Italy \\ E-mail: polina.zemkodstudenti.unipd.it
}

I summarize the main observational properties of nova-like (NL) systems in a wide energy range, from the radio to the X-rays and discuss them in the framework of the theoretical models. The current classification of NLs and the defining characteristics of each sub-type are discussed. I also present a statistical overview of a number of the known NL systems of different sub-types and compare their orbital period distribution with that of dwarf novae and novae.

The Golden Age of Cataclysmic Variables and Related Objects - III, Golden2015

7-12 September 2015

Palermo, Italy

\footnotetext{
* Speaker.
} 


\section{Introduction}

Nova-like (NL) stars are a group of CVs characterised by the absence of DN outbursts. The absence of DN outbursts indicates that the accretion disk in a NL star is in a stable, hot state, which prevents formation of the thermal-viscous instability. This requires a significantly high mass transfer rate of $\sim 10^{-8} \mathrm{M}_{\odot} \mathrm{yr}^{-1}$, which was indeed confirmed observationally and is also supported by the temperature estimates of the WDs [1], [2]. On the other hand, accretion disks in NLs do not perfectly match the standard stable disk scenario (when the mass accretion rate is constant throughout the disc). Thus Rutten et al. [3] found that the radial temperature dependence in the inner regions of accretion disks in several NLs is flatter than the predicted $\mathrm{T} \sim \mathrm{R}^{-3 / 4}[4,3]$. This may indicate that the central regions of the disks release part of the accretion energy in a nonradiative form. A departure from the canonical steady-state profile was found also in the infrared (IR) and can be caused by bremsstrahlung emission or circumbinary dust [5].

According to the International Variable Star Index (VSX) ${ }^{1}$, there are 136 NL systems, which can be subdivided into several groups according to their observational properties. The existing classification of NLs is somewhat tricky since different types of NLs are defined according to different criteria, spectroscopic ones for the SW Sex-type NLs, photometric ones for the VY Scl type, and the X-ray properties for the V Sge type, and there are significant overlaps of characteristics. In addition, there are NLs that have never been observed to show specific properties of the aforementioned groups and are called UX UMa-type NLs.

Fig. 1 shows the orbital period distribution of CVs of different types. The top panel represents non-magnetic DNe (light grey), novae (red hatched), NLs (green) and the total number of these objects (white). The grey hatched region between 2 and $3 \mathrm{~h}$ shows the period gap. NLs are clustered near the right edge of the period gap, and are the dominant population in the period range of 3-3.5 h. The bottom panel shows the orbital period distribution of NLs of different types. The white columns represent the total number of NLs, the green are UX UMa-type NLs, the black hatched are VY Scl-type stars (excluding those that also belong to the SW Sex-type), the purple are SW Sex, including those with low states, the blue hatched are NLs with magnetic WDs (intermediate polars), and the red hatched are V Sge-type NLs. The list of the non-magnetic DNe, novae, VY Scl- and UX UMa-type NLs was taken from the Catalogue of cataclysmic binaries, low-mass Xray binaries and related objects (7th Edition, Release 7.21, March 2014) [6]. For the SW Sex-type NLs we referred to the The Big List of SW Sextantis Stars (v5.1.1 - May 2015) [7] ${ }^{2}$. The list of V Sge-type stars is presented in [8]. NL/IPs are LS Peg [9], V795 Her [10] and DW Cnc [11]. In total, I plotted 98 NLs (32 of the UX UMa type, 31 of the VY Scl type, 29 of the SW Sex type, including 12 with low states, 3 IPs and 4 of the V Sge type)

\subsection{VY Scl-type stars and their low states}

Some NLs show so-called low states, which are sudden drops of optical magnitude by more than 1.5 mag on a time-scale of less than 150 days [12]. So far, about 30 NLs with low sates are known (see the VSX catalog and also [6]). These low states can be as deep as 7 mag and last for years (e.g. the low states in TT Ari). The drop of luminosity is associated with a sudden

\footnotetext{
${ }^{1}$ https://www.aavso.org/vsx/

${ }^{2}$ See D. W. Hoard's Big List of SW Sextantis Stars at http://www.dwhoard.com/biglist.
} 


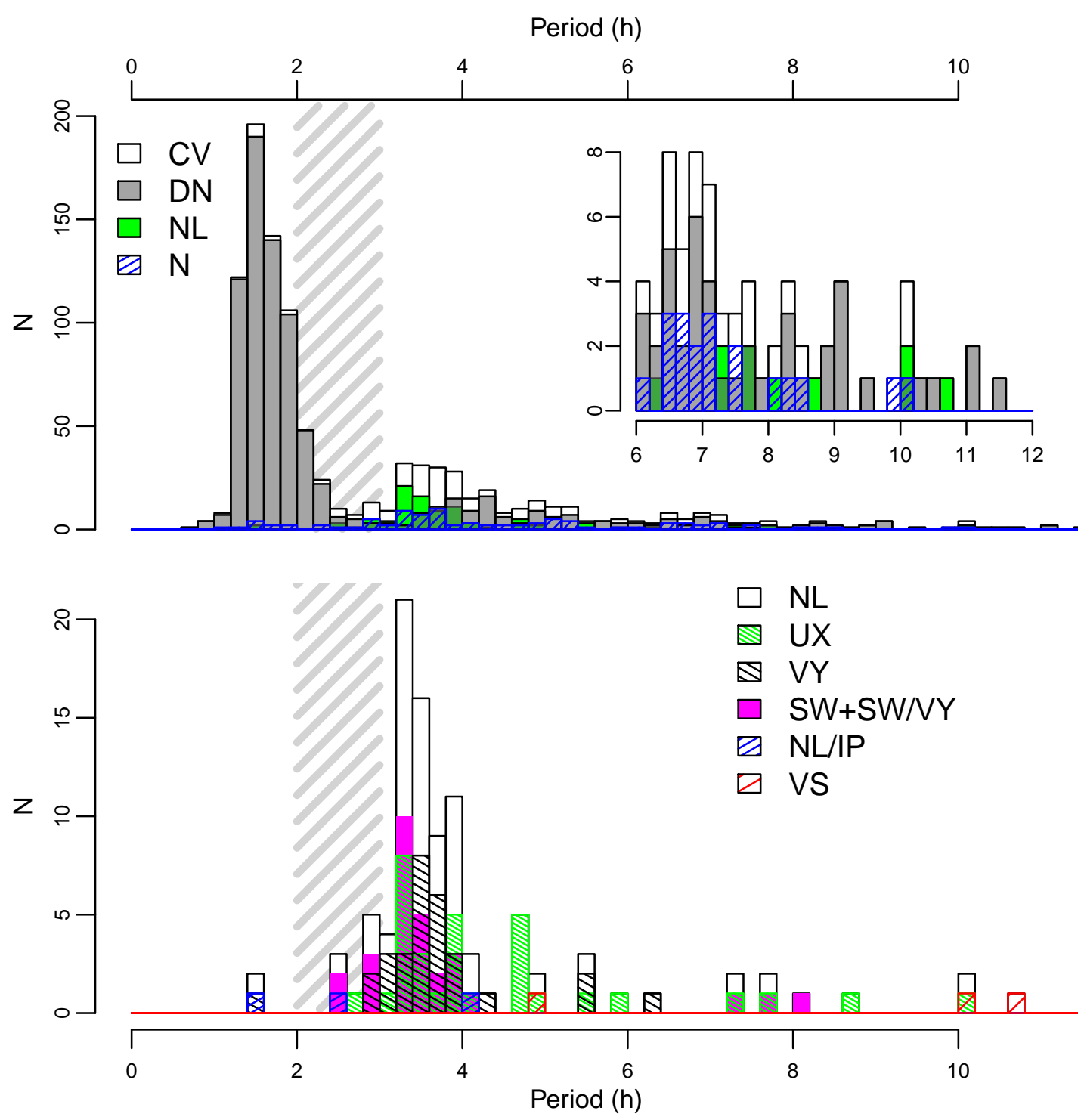

Figure 1: The orbital period distribution of CVs of different types. Top: non-magnetic DNe (light grey), novae (blue hatched), NLs (green) and the total number of these objects (white). Bottom: The orbital period distribution of NLs of different types. The total number of NLs (white), UX UMa-type NLs (green hatched), VY Scl-type stars, excluding those, that also belong to the SW Sex-type (black hatched), SW Sex including those with low states (purple), NLs with magnetic WDs (blue hatched), and V Sge-type NLs (red hatched).

decrease of the mass transfer rate from the secondary. Two explanations of this phenomenon have been proposed: the appearance of star spots on the surface of the secondary drifting around the L1 point [13] and shielding of the WD radiation by the accretion disk, which can suppress the irradiation-driven mass transfer [14]. The secondary's stellar spots scenario is the most widely accepted. It can also explain the shape of light curves during the transition from the high to the low state. [12] noticed that about $40 \%$ of the low states from their sample had dual slopes (transitions occurred in two steps, from a high to intermediate and then from the intermediate to a low state) while the rest had single slopes. This distribution is consistent with that of the diameters of umbra 
and penumbra regions in a typical sunspot and indicates that dual slopes arise from crossing of both star spot umbra and penumbra, whereas single slopes arise from crossing only the penumbra region. However, low states can last for years and it is not clear what can make a star spot so stable and hold it around one place for such a long time.

The low states in NLs are very intriguing for several reasons. Although a decrease of the mass transfer rate from the secondary should move the accretion disk from the stable and hot state to the zone of the DN instability, no DN outbursts were observed neither during low nor intermediate states [15]. Even if the mass transfer rate is switched off, there should be enough material left in the accretion disk for several dwarf nova eruptions. Leach et al. [16] proposed that a moderately hot WD $(40000 \mathrm{~K})$ can irradiate the accretion disk, preventing the thermal-viscous instability, however this requires fine tuning [17]. Another explanation involves a magnetic WD, whose magnetosphere truncates the inner region of the accretion disk, preventing "inside-out" outbursts [17]. In fact, both these scenarios can work. Far ultraviolet (FUV) observations indeed show that the WDs in NLs have temperatures of $33000-50000 \mathrm{~K}[1,2]$ and there are also several indications that these systems are weakly magnetic ${ }^{3}$ (see also Section 4 ).

Another question to answer is what happens with the accretion disks during low states. Some eclipsing NLs show shrinking of the accretion disks (see e.g. [18] for DW UMa), while other systems do not [19]. [20] attempted to measure the accretion disk radius change in TT Ari during its last transition to the low state following the period of negative superhumps, and showed that the accretion disk shrank by at least $15 \%$.

The low states are also important for the CV evolution. The high state accretion disk is the dominant source of light in a NL system, outshining both binary components. Only during the low states can the WD be observed directly, allowing a measurement of its surface effective temperature and the long-term mass transfer rate.

\subsection{SW Sex-type stars}

The group of SW Sex-type stars is distinguished by their spectroscopic characteristics. Originally, SW Sex-type stars were defined as eclipsing binary systems, which show single-peak emission lines in their spectra in contrast to what is expected from a highly inclined accretion disk in Keplerian rotation [21]. These objects also show strong He II $\lambda 4686$ emission, high velocity line wings and transient absorption features at orbital phase 0.5 [21]. Although single-peak emission lines are the most important characteristics of SW Sex stars, in the most current classification SW Sex are not only systems with high orbital inclination. The second classification criterium is an orbital phase offset of $0.1-0.2$ cycles in the emission-line radial velocity curves with respect to the photometric ephemeris [22]. So far there are 30 confirmed SW Sex-type NLs and 40 more candidates $^{4}$. 12 out of 30 confirmed SW Sex members also belong to the group of VY Scl-type stars since they undergo low states.

\section{$1.3 \mathrm{~V}$ Sge}

The least numerous and the most puzzling group of NLs are the V Sge-type stars. Steiner\&Diaz [23] proposed that these stars have very high mass transfer rate $\left(\sim 10^{-7} \mathrm{M}_{\odot} \mathrm{yr}^{-1}\right)$, occurring on a

\footnotetext{
${ }^{3}$ The Magnetic Scenario for the SW Sextantis Stars http://www.dwhoard.com/biglist/magnetic.

${ }^{4}$ See D. W. Hoard's Big List of SW Sextantis Stars at http://www.dwhoard.com/biglist [7]).
} 
thermal time-scale, which results in a hydrostatic nuclear burning on the surfaces of their WDs. Such hydrogen burning would result in luminous soft X-ray emission, which is not observed, but this may be because of the intrinsic (wind) and interstellar absorption (for more details see [23] and [24]). There are three known members of the V Sge group apart from the prototype itself: V617 Sgr, WX Cen and QU Car (the latter is a candidate) [8]. Their defining properties are high-ionization emission lines of $\mathrm{O} V I$ and $\mathrm{N} V$, the equivalent widths ratio $\mathrm{EW}$ (He II 4686 A) $/ \mathrm{EW}(\mathrm{H} \beta)>2$, the lack or weakness of He I lines and P Cyg profiles, which is a signature of a strong wind. Compared with the orbital periods of other NLs, those of V Sge-type stars are long: $\sim 5-12 \mathrm{~h}$ (see also fig. 1). The orbital light curves are either low-amplitude sinusoidal or highamplitude asymmetric, with primary and secondary eclipses. They also show high/low states, with amplitudes smaller than those of VY Scl-type stars. The atmospheric absorption features from the secondary are not observed in these systems.

\section{Ultraviolet observations and the evolution status of NLs}

FUV observations allow an estimate of the WD temperature in a NL, which correlates with the time-averaged accretion rate. Many UV and FUV observations have shown that the WDs in NLs can be as hot as $50000 \mathrm{~K}$. [25] plotted the $\mathrm{T}_{\text {eff }}-\mathrm{P}_{\text {orb }}$ diagram and found that NLs do not fit the general trend of non-magnetic CVs in the same range of the orbital periods and have higher temperatures than what is expected from accretion driven by magnetic braking. The authors proposed that NLs represent an exceptional stage of CV evolution and were probably born with the $3-4 \mathrm{~h}$ orbital periods. However, [26] noticed that almost all eclipsing CVs in this period range are SW Sex stars and suggested that CVs crossing this $3-4 \mathrm{~h}$ stripe during their evolution from longer to shorter orbital periods can show properties of SW Sex stars. The fact that old novae with the same range of orbital periods have much lower mass transfer rate and spectroscopic characteristics similar to those of DNe rather than those of NLs may be the result of the response of the secondary to the nova explosion (a variation of the hibernation model) [27]. So far, there are only two old novae (BT Mon and V533 Her) among the confirmed SW Sex stars and V630 Sgr is a nova and VY Scl-type candidate [28]. Mass transfer rates in NLs are closer to those of recurrent novae, but NLs do not undergo recurrent nova explosions.

Another possible missing link lies between NLs and ER UMa-type DNe ${ }^{5}$. [29], [30] detected increasing trends in the supercycle lengths (time interval between two subsequent superoutbursts) of ER UMa-type stars, which implies a gradual decrease of the mass transfer rates in these systems. If these trends existed also in the past, it would mean that ER UMa-type stars were behaving like NLs 100-200 years ago (see fig.1 from [31]). Therefore, BK Lyn, the object that was caught at the time of transition from a NL to ER UMa-like behaviour may be not the only one example. However, in case of BK Lyn, the time scale of the mass transfer rate change was much lower.

\section{X-ray observations and surface hydrogen burning}

Although the high mass transfer rate in NLs should make them significantly bright in X-rays,

\footnotetext{
${ }^{5}$ ER UMa-type stars are a sub-type of SU UMa-type DNe. They show outbursts together with a larger amplitude superoutbursts with very short recurrence times, indicating the highest mass transfer rate among DNe.
} 
many SW Sex-type stars are faint or not detected at all. This can be due to the intrinsic absorption, which is especially important for the soft X-rays (see [32] and the ROSAT All Sky Survey). Since SW Sex stars are mostly high inclination systems, they can also be faint in X-rays due to absorption by the accretion disk, which is viewed almost edge-on.

On the other hand, several VY Scl-type stars have been detected in X-rays even with short Swift exposures during their low states. The observed X-ray spectra of SW Sex and VY Scl-type stars can be well fitted either with the cooling flow model or with two-temperature thermal plasma emission model, in which the highest temperature is about $5-10 \mathrm{keV}$, typical of accreting nonmagnetic WDs [32], [33], [34]. X-ray grating observations of TT Ari showed that there were two different X-ray emitting regions: one related to accretion and the other probably to wind from the binary system [33].

X-ray observations of NLs rised a question about possibility of hydrogen burning on the surfaces of the WDs in these systems. Greiner et al. [35] found an anti-correlation in the optical and X-ray intensity in a VY Scl-type star - V751 Cyg — and proposed that this object can burn hydrogen quietly during low states. [36] and [37] also suggested that thermonuclear burning occurs in the low states of V Sge and BZ Cam, implying that they may periodically become super soft $\mathrm{X}$-ray sources. The hydrogen burning scenario was also supported by the observations in other wavelengths. Hachisu\&Kato [38] explained the orbital curves and high/low state transitions of V Sge with an optically thick, massive wind, completely obscuring the soft X-rays. Honeycutt [39] noticed that during the high states many NLs show so-called "stunted" outbursts, which may be normal thermal instabilities in the accretion disk occurring at steady background luminosity, resulting from nuclear burning on the WD surface. Patterson et al. [24] found that V Sge is optically very luminous and there must be a strong ionizing source in the system. Moreover, a hot hydrogen burning WD can heat the accretion disk and prevent the thermal instability, explaining the absence of DN outbursts in VY Scl-type stars. If the WDs in NLs burn hydrogen quietly without triggering a thermonuclear runaway, these objects are of particular importance for the evolution since they can reach the Chandrasekhar mass and the conditions for type Ia supernovae outbursts.

However, the X-ray observations of both V751 Cyg and V Sge, performed with the ROSAT High Resolution Imager (HRI) ${ }^{6}$, did not offer the spectral resolution that is necessary to draw conclusions on the hardness of the X-ray spectrum. In more recent years an X-ray observation of the VY Scl system V504 Cyg in the low state failed to reveal a luminous SSS [40]. Zemko et al. [33] analysed all availiable archival X-ray and UV observations of four VY Scl-type stars in both high and low state and also did not detect the proposed supersoft emission.

\section{Emission lines flaring and WD magnetic field}

Many SW Sex-type stars show emission line flaring, that is a modulation of the fluxes and equivalent widths of emission lines. This phenomenon is very often observed in IPs, because of the dynamics of the line-emitting source (see [41] and references therein) and occurs on a timescale of tens of minutes. QPOs observed in the X-rays in SW Sex- and VY Scl-type stars have the same characteristic periods of $\sim 1000-2000$ s. Patterson et al. [42] noticed that this timescale is exactly

\footnotetext{
${ }^{6}$ ROSAT HRI has a high time resolution but negligible energy resolution.
} 
the expected WD rotation period in a magnetic system in spin equilibrium with the orbital period of 3-4 h. and proposed that SW Sex-type stars can be a borderline polars and that the very high mass transfer rate may "drown" their magnetic properties.

Interestingly, Rodriguez-Gil et al. [43] found significant variability of the optical spectra of BB Dor, which is a SW Sex- and VY Scl-type star, during its low state and interpreted it as a result of accretion events on a timescale of tens of minutes. The source of this extra emission was located somewhere between two stars and was either related to the material transferred from the secondary to the magnetosphere of the WD in the absence of an accretion disc, or to the hot spot region in the outer rim of a cold, remnant accretion disc. The first possibility assumes that BB Dor hosts magnetic WDs and behaves like an IP during high states and like disk-less polars during low states. A similar variability of the optical spectra was observed in TT Ari during its last low state (Zemko et al. in prep.). In addition, TT Ari showed outburst-like events in the optical band with amplitudes up to $2.5 \mathrm{mag}$. on a timescale of $2-3 \mathrm{~h}$, possibly related to sporadic accretion events. Although the magnetic scenario has some observational and theoretical support, there are only three confirmed IPs among NLs. Two of them are SW Sex-type stars, in which circular polarization was detected (LS Peg [9] and V795 Her [10]), and the third one, DW Cnc, shows an X-ray modulation associated with the WD spin period [11].

\section{Conclusions}

NL stars are luminous in a wide energy range, which makes possible their multi-wavelength observations. I summarize the most important problems of the broad band studies of NL systems, from the radio to the X-rays.

- Radio emission was detected in four NLs (V3885 Sgr [45], RW Sex, V603 Aql, TT Ari [44]). In the radio range flares and other variability were observed, and circular polarization was measured. However, the root causes of these phenomena have not been established and further observations are needed [44].

- There is no uniform theory explaining the observational properties of the SW Sex-type stars. Some features can be understood assuming a strong magnetic field (like that of IPs), but it would imply that just above the period gap, where the SW Sex-type stars dominate, CVs are mostly magnetic, while below it, where the majority of DNe are found, CVs are nonmagnetic.

- Although it is now clear that NLs are a numerous class of objects and are the dominant population in the $3-3.5 \mathrm{~h}$ range of the orbital periods, their place in the overall $\mathrm{CV}$ evolution is still uncertain. The WD temperatures in NLs are higher than is expected from mass transfer rate driven by the magnetic braking [25]. Although this conclusion was based on a sample of only three objects. We need further UV and FUV observations of NLs during low states in order to measure the WD effective temperatures and to explore their evolution status.

- It is still not known whether WDs in NLs experience non-explosive hydrogen burning. The original idea was based on low-resolution X-ray observations and since then there was no 
observational confirmation of the proposed supersoft emission. X-ray observations of the V Sge-type stars and particularly of the prototype itself with the currently operating X-ray satellites are required in order to prove or disprove the hydrogen burning scenario.

\section{References}

[1] Hoard, D. W., Linnell, A. P., Szkody, P., et al. 2004, ApJ, 604, 346

[2] Gänsicke, B. T., Sion, E. M., Beuermann, K., et al. 1999, A\&A, 347, 178

[3] Rutten, R. G. M., van Paradijs, J., \& Tinbergen, J. 1992, A\&A, 260, 213

[4] Bath, G. T., \& Pringle, J. E. 1981, MNRAS, 194, 967

[5] Hoard, D. W., Long, K. S., Howell, S. B., et al. 2014, ApJ, 786, 68

[6] Ritter, H., \& Kolb, U. 2003, A\&A, 404, 301

[7] Hoard, D. W., Szkody, P., Froning, C. S., Long, K. S., \& Knigge, C. 2003, AJ, 126, 2473

[8] Oliveira, A. S., Lima, H. J. F., Steiner, J. E., Borges, B. W., \& Cieslinski, D. 2014, MNRAS, 444, 2692

[9] Rodríguez-Gil, P., Casares, J., Martínez-Pais, I. G., Hakala, P., \& Steeghs, D. 2001, ApJl, 548, L49

[10] Rodríguez-Gil, P., Casares, J., Martínez-Pais, I. G., \& Hakala, P. J. 2002, in Astronomical Society of the Pacific Conference Series, Vol. 261, The Physics of Cataclysmic Variables and Related Objects, ed. B. T. Gänsicke, K. Beuermann, \& K. Reinsch, 533

[11] Patterson, J., Thorstensen, J. R., Vanmunster, T., et al. 2004, PASP, 116, 516

[12] Honeycutt, R. K., \& Kafka, S. 2004, AJ, 128, 1279

[13] Livio, M., \& Pringle, J. E. 1994, ApJ, 427, 956

[14] Wu, K., Wickramasinghe, D. T., \& Warner, B. 1995, Publ. Astron. Soc. Australia, 12, 60

[15] King, A. R., \& Cannizzo, J. K. 1998, ApJ, 499, 348

[16] Leach, R., Hessman, F. V., King, A. R., Stehle, R., \& Mattei, J. 1999, MNRAS, 305, 225

[17] Lasota, J.-P., \& Hameury, J.-M. 2004, in Astronomical Society of the Pacific Conference Series, Vol. 315, IAU Colloq. 190: Magnetic Cataclysmic Variables, ed. S. Vrielmann \& M. Cropper, 46

[18] Stanishev, V., Kraicheva, Z., Boffin, H. M. J., et al. 2004, A\&A, 416, 1057

[19] Shears, J., Gaensicke, B., Rodriguez-Gil, P., et al. 2015, ArXiv e-prints, arXiv:1503.07992

[20] Zemko, P., \& Kato, T. 2014, Contributions of the Astronomical Observatory Skalnate Pleso, 43,487

[21] Thorstensen, J. R., Ringwald, F. A., Wade, R. A., Schmidt, G. D., \& Norsworthy, J. E. 1991, AJ, 102, 272

[22] Rodríguez-Gil, P., Schmidtobreick, L., \& Gänsicke, B. T. 2007a, MNRAS, 374, 1359

[23] Steiner, J. E., \& Diaz, M. P. 1998, PASP, 110, 276

[24] Patterson, J., Kemp, J., Shambrook, A., et al. 1998, PASP, 110, 380

[25] Townsley, D. M., \& Gänsicke, B. T. 2009, ApJ, 693, 1007

[26] Schmidtobreick, L. 2013, Central European Astrophysical Bulletin, 37, 361 
[27] Schmidtobreick, L., \& Tappert, C. 2014, in Astronomical Society of the Pacific Conference Series, Vol. 490, Stell Novae: Past and Future Decades, ed. P. A. Woudt \& V. A. R. M. Ribeiro, 29

[28] Mróz, P., Udalski, A., Poleski, R., et al. 2015, ApJS, 219, 26

[29] Zemko, P., Kato, T., \& Shugarov, S. Y. 2013, PASJ, 65, 54

[30] Otulakowska-Hypka, M., \& Olech, A. 2013, MNRAS, 433, 1338

[31] Osaki, Y. 1995, PASJ, 47, L11

[32] Hoard, D. W., Lu, T.-N., Knigge, C., et al. 2010, AJ, 140, 1313

[33] Zemko, P., Orio, M., Mukai, K., \& Shugarov, S. 2014, MNRAS, 445, 869

[34] Page, K. L., Osborne, J. P., Beardmore, A. P., et al. 2014, A\&A, 570, A37

[35] Greiner, J., Tovmassian, G. H., Di Stefano, R., et al. 1999, A\&A, 343, 183

[36] Greiner, J., \& Teeseling, A. 1998, A\&A, 339, L21

[37] Greiner, J., Tovmassian, G., Orio, M., wt al., 2001, A\&A,376, 1031

[38] Hachisu, I., \& Kato, M. 2003, ApJ, 598, 527

[39] Honeycutt, R. K. 2001, PASP, 113, 473

[40] Greiner, J., Schwarz, R., Tappert, C., et al., 2010, Astronomische Nachrichten, 331, 227

[41] Rodríguez-Gil, P., Gänsicke, B. T., Hagen, H.-J., et al. 2007b, MNRAS, 377, 1747

[42] Patterson, J., Fenton, W. H., Thorstensen, J. R., et al. 2002, PASP, 114, 1364

[43] Rodríguez-Gil, P., Schmidtobreick, L., Long, K. S., et al. 2012, MNRAS, 422, 2332

[44] Coppejans, D. L., Körding, E. G., Miller-Jones, J. C. A., et al. 2015, MNRAS, 451, 3801

[45] Körding, E. G., Knigge, C., Tzioumis, et al., 2011, MNRAS, 418, L129 